\title{
Is Blood Urea Concentration an Independent Predictor of Positive Endoscopic Findings in Presumed Upper Gastrointestinal Bleeding?
}

\author{
Deepti Chopra Morgan Rosenberg Paul Moayyedi Neeraj Narula \\ Division of Gastroenterology, Department of Medicine, McMaster University, Hamilton, ON, USA
}

\begin{abstract}
Keywords
Urea $\cdot$ Upper gastrointestinal bleeding $\cdot$ High-risk .

Endoscopy
\end{abstract}

\begin{abstract}
Background: The test characteristics of blood urea concentration in the identification of upper gastrointestinal bleeding (UGIB) or high-risk endoscopic lesions have not been clearly determined. This study aimed to elucidate if urea independently correlates with the presence of positive endoscopic findings in cases of presumed UGIB and understand the diagnostic value of this parameter when assessing a patient with potential UGIB. Methods: A retrospective cohort study was conducted at Hamilton Health Sciences hospitals examining patients who had upper endoscopy for presumed UGIB. Contingency tables were generated to determine the test characteristics of urea at different thresholds for prediction of UGIB. A crude OR was calculated for odds of bleeding being identified on endoscopy based on varying thresholds of urea, and adjusted ORs were calculated using logistic regression modelling. Results: Variables significantly associated with detecting a source of Gl bleeding at endoscopy included increase in urea (OR 1.06, 95\% Cl 1.011.09), male gender (OR $2.02,95 \% \mathrm{Cl} 1.08-3.77)$, presence of melena (OR 2.37, 95\% Cl 1.06-5.33), and hematemesis (OR $3.88,95 \% \mathrm{Cl} 1.70-8.83$ ), when adjusted for other covariates. The odds of identifying UGIB at endoscopy in patients with urea $\geq 10 \mathrm{mmol} / \mathrm{L}$ was $3.73(95 \% \mathrm{Cl} 1.90-7.31)$ times higher
\end{abstract}

\section{KARGER}

(c) 2019 S. Karger AG, Basel

E-Mail karger@karger.com

www.karger.com/ddi than for patients with urea $<10 \mathrm{mmol} / \mathrm{L}$. Conclusion: Urea level is an independent predictor of positive endoscopic findings in presumed UGIB, and urea $\geq 10 \mathrm{mmol} / \mathrm{L}$ may be a useful threshold to help guide clinicians towards clinically significant bleeding that could warrant early endoscopic evaluation.

(c) 2019 S. Karger AG, Basel

\section{Introduction}

Acute upper gastrointestinal bleeding (UGIB) is associated with significant morbidity, mortality, and cost to healthcare system [1]. The mortality rate of UGIB has remained steady at 5-10\% despite advances in medical, endoscopic, and surgical therapies [1]. The estimated annual incidence of acute UGIB in both North America and the United Kingdom is between 100 and 200 per 100,000 population [2-4]. Roughly one third of patients with bleeding peptic ulcer disease will develop recurrent bleeding in the following 1-2 years and 40-50\% will re-bleed within the subsequent 10 years if left untreated after initial healing $[5,6]$. Esophageal varices account for $<10 \%$ of all causes of UGIB. However, patients with variceal hemorrhage have a mortality rate of at least 30\% during their initial hospitalization and a 1 year mortality rate approaching 60\% [7]. Patients who have bled once from esophageal varices have a $70 \%$ chance of re-bleeding, and almost $30 \%$ of further bleeding episodes are fatal [8]. 
Not all cases of presumed UGIB based on clinical presentation have endoscopic findings consistent with UGIB or result in endoscopic intervention at the time of esophagogastroduodenoscopy (EGD). High-risk endoscopic lesions are important in the management of patients with UGIB as they portend a higher risk of re-bleeding and result in additional hospitalization for medical management [1]. Predicting patients who may have high-risk lesions is important for triaging cases requiring emergent endoscopy. EGD is effective in diagnosing and treating most causes of UGIB and is associated with reduced blood transfusions and hospital/intensive care length of stay, particularly if endoscopy occurs within $24 \mathrm{~h}$ of hospital admission [9-11].

Several prediction rules have been developed for use in UGIB, including the Blatchford score, which uses blood urea concentration as 1 of 8 prognostic variables, because to date, no single clinical or biochemical factor is diagnostic of UGIB severity $[11,12]$. The Blatchford score is calculated by assigning a score to the following component values: blood urea, hemoglobin, systolic blood pressure, heart rate, melena, syncope, hepatic disease, and cardiac failure. A Blatchford score $>0$ was $99-100 \%$ sensitive for identifying a severe bleed in several studies and performs better than the Rockall score [11-17]. The Blatchford score requires multiple variables for calculation, however. It would be useful if a single test could help predict the risk of true UGIB. Although the Blatchford score incorporates urea level within its score, the test characteristics of urea concentration alone in the identification of UGIB has not been clearly determined and current literature on the topic is sparse and controversial, even suggesting that the initial urea level is not helpful in identifying patients with high-risk lesions [18]. Some scores, such as the Rockall and AIMS65, do not incorporate urea into their calculations. We therefore aimed to evaluate the predictive value of urea, particularly assessing its utility in identifying a lesion on EGD that could reasonably account for UGIB.

\section{Methods}

\section{Study Design}

We performed a retrospective cohort study of adult patients undergoing inpatient EGD for presumed UGIB at Hamilton Health Sciences hospitals in Ontario, Canada. We searched for all endoscopic procedures performed by gastroenterologists at Juravinski Hospital, Hamilton General Hospital, and McMaster University Medical Centre from September 15, 2009 to 2012. Patients were included in the study if the indication for EGD was stated as melena, hematemesis, UGIB, or gastrointestinal bleed- ing of unknown source. Medical charts of included patients were also accessed to obtain information on patient demographic data and other relevant clinical characteristics. Patients with no available serum urea were excluded. Ethics approval for this study was obtained from the Hamilton integrated Research Ethics Board.

\section{Patient Characteristics}

Patient demographics were collected for all patients included in the study. Looking back 4 days from the time of endoscopy (to account for weekends), the following information was recorded: urea level at the time of referral to gastroenterology for GI bleeding, lowest hemoglobin, whether transfusion of packed red blood cells occurred, signs and symptoms of gastrointestinal bleeding, serum creatinine, serum prothrombin time - international normalized ratio, platelet count, use of low dose aspirin, other nonsteroidal anti-inflammatories (NSAIDS), or therapeutic anti-coagulation, and if the patient was admitted to the intensive care unit (ICU). The typical length of time from urea level determination (at the time of referral to gastroenterology) to endoscopy was 24-48 h. When available, the baseline urea prior to suspected bleeding event was obtained. Use of endoscopic intervention at the time of EGD with argon plasma coagulation, hemoclips, epinephrine injection, cautery, or variceal banding was also recorded. Dictated Blatchford scores from the gastroenterology consult notes were recorded when available, otherwise the score was calculated from emergency room triage vital signs and clinical chart information.

\section{Outcome Assessment}

A positive EGD was defined as one with findings that could reasonably account for an episode of UGIB including esophagitis (LA Grade C or higher), presence of medium or large varices, Mallory-Weiss tear, $>5$ gastric erosions, any ulcer, suspected malignancies (i.e., ulcerated mass), gastric antral vascular ectasia, Dieulafoy lesion, angiodysplasia with evidence of active bleeding, severe portal hypertensive gastropathy, and aortoenteric fistula. Lesions were stratified as low- or high-risk lesions. High-risk lesions were ulcers with Forrest Classification IIA or higher, varices $>5 \mathrm{~mm}$ or with high-risk stigmata (e.g., red wale sign), and aortoenteric fistula. All others were assigned to the low-risk category.

\section{Analysis}

Factors thought to influence likelihood of identifying a source of UGIB on EGD were determined a priori based on previous knowledge. Contingency tables were generated to determine the test characteristics of urea at different thresholds for prediction of UGIB sources at EGD. Data for all patients with UGIB on EGD, and for patients with high-risk UGIB lesions on EGD, were compared to patients without UGIB identified on EGD using the Mann-Whitney test, as data were not normally distributed. Blatchford scores were compared for patients with different urea levels using a scatterplot and the Spearman coefficient was calculated. Receiver operating characteristic curves were also studied to determine accuracy. Sensitivity, specificity, positive predictive value (PPV) and negative predictive value (NPV) of urea at different thresholds for identifying a lesion on EGD was calculated. Additionally, several subgroups were selected to assess if urea was predictive in specific patient populations, including NSAID use, symptoms of hematemesis and melena, presence of cirrhosis, those with high-risk bleeding sourc- 
Table 1. Study population demographics

\begin{tabular}{lc}
\hline Characteristic & $n(\%), n=261$ \\
\hline Age, years, mean \pm SD & $70.15 \pm 15.02$ \\
Gender & $145(55.56)$ \\
$\quad$ Male & $116(44.44)$ \\
$\quad$ Female & $28(10.72)$ \\
Cirrhosis & $13.62 \pm 10.51$ \\
Urea at the time of referral (normal range 2.3-7.4), mmol/L, mean $\pm \mathrm{SD}$ & $111.87 \pm 90.51$ \\
Creatinine (normal range 64-111), $\mu$ mol/L, mean $\pm \mathrm{SD}$ & $0.13 \pm 0.07$ \\
Urea:creatinine, mean \pm SD & $1.71 \pm 2.31$ \\
INR (normal range 0.8-1.2), mean \pm SD & $85.93 \pm 22.84$ \\
Hemoglobin (normal range 130-180), g/L, mean \pm SD & $23(8.81)$ \\
ICU admission & $163(62.45)$ \\
Transfused & $190(72.80)$ \\
Melena & $95(36.40)$ \\
Hematemesis & $103(39.46)$ \\
ASA use & $20(7.66)$ \\
Plavix use & $116(44.44)$ \\
Anticoagulation use & $81(31.03)$ \\
NSAID use & $44(16.86)$ \\
\hline
\end{tabular}

ICU, intensive care unit; NSAID, non-steroidal anti-inflammatories; ASA, aspirin; INR; prothrombin time international normalized ratio.

es, or those who had intervention at EGD. We also examined if urea was useful for identifying a source of GI bleeding at EGD in patients with normal renal function, as those with abnormal renal function could have elevated urea for reasons other than GI bleeding. Blatchford scores were also compared between patients with different urea thresholds. Where applicable, 95\% CIs were provided.

A crude OR was calculated for odds of identifying a source of GI bleeding on endoscopy. Odds of identifying a GI bleeding source based on thresholds of urea $\geq 5,7.5,10,12.5,15$, and 20 $\mathrm{mmol} / \mathrm{L}$, were compared to odds lower than these. Adjusted ORs were then calculated using logistic regression to account for the factors that were determined a priori. Covariates included in the model were age, sex, hemoglobin, presence of melena, presence of hematemesis, admission in ICU, and use of aspirin, warfarin, clopidogrel, or NSAIDS. All analyses were performed using GraphPad Prism (version 5.03, GraphPad Software, San Diego, CA, USA) and SAS 9.4 (Cary, NC, USA).

\section{Results}

A total of 261 patients were included. The study population had a mean age of 70.15 years. Of the included patients, $55.56 \%$ were male, $10.72 \%$ of patients had cirrhosis, $44.44 \%$ were on anticoagulants, $47.12 \%$ were on antiplatelet agents, and $31.03 \%$ were NSAID users. The mean urea level at the time of referral to gastroenterology was
$13.62 \mathrm{mmol} / \mathrm{L}(\mathrm{SD} \pm 10.51)$. As all patients had serum urea data available, no patients were excluded due to lack of information. Complete patient demographic characteristics are given in Table 1.

In an adjusted multivariate logistic regression analysis, urea level was significantly associated with increased odds of identifying a source of bleeding at UGIB. Each unit increase in urea increased the odds of identifying UGIB at EGD by 1.06 (95\% CI 1.01-1.09) times, when adjusted for other covariates. Three additional covariates were significantly associated with increased odds of positive EGD findings from the multivariate regression analysis: male gender (OR 2.02, 95\% CI 1.08-3.77), presence of melena (OR 2.37, 95\% CI 1.06-5.33), and presence of hematemesis (OR 3.88, 95\% CI 1.70-8.83), all adjusted for other covariates (Table 2). There was no interaction between variables.

The OR of identifying a source of UGIB on EGD at different thresholds of urea is given in Table 3. The highest adjusted OR was seen using a threshold of urea $\geq 10$ $\mathrm{mmol} / \mathrm{L}$. The odds of identifying a source of UGIB at endoscopy for patients with urea $\geq 10 \mathrm{mmol} / \mathrm{L}$ was 3.73 (95\% CI 1.90-7.31) times higher than for patients with urea $<10 \mathrm{mmol} / \mathrm{L}$. The median urea level in patients with UGIB sources compared to those without UGIB sources was 11.3 vs. $7.9 \mathrm{mmol} / \mathrm{L}, p<0.0003$. The median urea 
Table 2. Results of multivariate regression analysis reporting associations of variables with risk of identifying a source of UGIB at endoscopy

\begin{tabular}{lcccc}
\hline Variable & Beta-coefficient & OR & $95 \%$ CI & $p$ value \\
\hline Urea at referral to GI & 0.0541 & 1.056 & $1.013-1.099$ & 0.0093 \\
Age, years & -0.0112 & 0.989 & $0.967-1.012$ & 0.3339 \\
Gender & 0.7034 & 2.021 & $1.084-3.765$ & 0.0267 \\
Hemoglobin & -0.00926 & 0.991 & $0.975-1.006$ & 0.2467 \\
Melena & 0.8637 & 2.372 & $1.055-5.331$ & 0.0366 \\
Hematemesis & 1.3552 & 3.878 & $1.702-8.833$ & 0.0013 \\
ASA & -0.1061 & 0.899 & $0.466-1.736$ & 0.7519 \\
Warfarin & -0.5875 & 0.556 & $0.269-1.149$ & 0.1128 \\
NSAIDS & 0.3449 & 1.412 & $0.845-2.358$ & 0.1876 \\
Plavix & 0.5258 & 1.692 & $0.481-5.945$ & 0.4122 \\
ICU & 0.1828 & 1.201 & $0.351-4.112$ & 0.7710 \\
Cirrhosis & 0.7312 & 2.077 & $0.648-6.661$ & 0.2187 \\
\hline
\end{tabular}

UGIB, upper gastrointestinal bleeding; NSAID, non-steroidal anti-inflammatories; ICU, intensive care unit; ASA, aspirin.

Table 3. ORs for urea thresholds to identify a source of UGIB at endoscopy

\begin{tabular}{|c|c|c|c|c|}
\hline Variable, $\mathrm{mmol} / \mathrm{L}$ & Crude ORs & Adjusted OR & $95 \% \mathrm{CI}$ & $p$ value \\
\hline Urea $\geq 5$ & 2.35 & 2.94 & $1.18-7.30$ & 0.02 \\
\hline Urea $\geq 7.5$ & 2.30 & 2.70 & $1.37-5.32$ & 0.004 \\
\hline Urea $\geq 10$ & 2.85 & 3.73 & $1.90-7.31$ & 0.0001 \\
\hline Urea $\geq 12.5$ & 2.27 & 2.52 & $1.29-4.96$ & 0.007 \\
\hline Urea $\geq 15$ & 2.70 & 3.36 & $1.55-7.29$ & 0.0022 \\
\hline Urea $\geq 20$ & 1.90 & 1.95 & $0.85-4.49$ & 0.12 \\
\hline
\end{tabular}

UGIB, upper gastrointestinal bleeding.

Table 4. Diagnostic performance of urea $\geq 10 \mathrm{mmol} / \mathrm{L}$ for identifying a source of UGIB at endoscopy

\begin{tabular}{lllllc}
\hline & Sensitivity & Specificity & PPV & NPV & LR \\
\hline Urea $\geq 10 \mathrm{mmol} / \mathrm{L}$ & 0.58 & 0.67 & 0.80 & 0.40 & 1.75 \\
\hline
\end{tabular}

UGIB, upper gastrointestinal bleeding; PPV, positive predictive value; NPV, negative predictive value.

level in those with a high-risk UGIB compared to nonbleeders was 14.0 vs. $7.9 \mathrm{mmol} / \mathrm{L}, p<0.0001$.

Since urea $\geq 10 \mathrm{mmol} / \mathrm{L}$ was the best threshold, we identified from our analysis in Table 3 that the utility of this threshold for identifying a source of GI bleeding on EGD was evaluated in various patient sub-populations. Overall, the PPV of urea $\geq 10 \mathrm{mmol} / \mathrm{L}$ for positive findings on EGD in our study population was $80 \%$ (Table 4), and the PPV of Blatchford scores at different cut-offs was 71-
$72 \%$ (Table 5). The PPV of urea $\geq 10 \mathrm{mmol} / \mathrm{L}$ for patients with normal renal function, cirrhosis, hematemesis, and melena was above $80 \%$. The PPV in patients using NSAIDS was $97 \%$. In patients who did not have high-risk bleeding sources or use of intervention at EGD, in most cases the urea was $<10 \mathrm{mmol} / \mathrm{L}$ (NPV of 90 and $85 \%$, respectively; Table 6).

Different thresholds of Blatchford scores were evaluated. Overall, these had low specificities for identifying 
Table 5. Diagnostic performance of Blatchford score in the study population

\begin{tabular}{llllll}
\hline Blatchford score & Sensitivity & Specificity & PPV & NPV & LR \\
\hline Blatchford $\geq 1$ & 0.99 & 0.03 & 0.71 & 0.67 & 1.02 \\
Blatchford $\geq 2$ & 0.99 & 0.07 & 0.71 & 0.56 & 1.05 \\
Blatchford $\geq 3$ & 0.98 & 0.11 & 0.72 & 0.67 & 1.09 \\
Blatchford $\geq 4$ & 0.96 & 0.12 & 0.72 & 0.56 & 1.09 \\
Blatchford $\geq 5$ & 0.94 & 0.13 & 0.72 & 0.48 & 1.08 \\
Blatchford $\geq 6$ & 0.92 & 0.17 & 0.72 & 0.46 & 1.11 \\
\hline
\end{tabular}

PPV, positive predictive value; NPV, negative predictive value.

Table 6. Diagnostic performance of urea $\geq 10 \mathrm{mmol} / \mathrm{L}$ for identifying a source of UGIB at endoscopy in various patient populations

\begin{tabular}{llllll}
\hline Patient population & Sensitivity & Specificity & PPV & NPV & LR \\
\hline Normal renal function & 0.38 & 0.81 & 0.80 & 0.40 & 2.00 \\
Cirrhosis & 0.58 & 0.50 & 0.88 & 0.17 & 1.17 \\
Hematemesis & 0.46 & 0.75 & 0.87 & 0.28 & 1.83 \\
Melena & 0.63 & 0.58 & 0.81 & 0.36 & 1.52 \\
NSAIDS & 0.65 & 0.93 & 0.97 & 0.43 & 9.04 \\
High-risk bleeding sources & 0.70 & 0.53 & 0.23 & 0.90 & 1.50 \\
Use of EGD intervention & 0.67 & 0.54 & 0.29 & 0.85 & 1.45 \\
\hline
\end{tabular}

UGIB, upper gastrointestinal bleeding; PPV, positive predictive value; NPV, negative predictive value; EGD, esophagogastroduodenoscopy.

a source of UGIB on EGD, for example, a Blatchford score of 1 or higher versus 0 was only $3 \%$ specific for identifying a source of UGIB on EGD but $99 \%$ sensitive (Table 5). A similar trend was observed when higher Blatchford score thresholds were evaluated. Among patients with urea $<5 \mathrm{mmol} / \mathrm{L}$, the median Blatchford score was 7 (interquartile range [IQR] 2-8), for urea of 5-10 $\mathrm{mmol} / \mathrm{L}$ the median score was 8 (IQR 6-10), for urea of $10-15 \mathrm{mmol} / \mathrm{L}$ the median score was 11 (IQR 10-13), and for urea $>15 \mathrm{mmol} / \mathrm{L}$ the median Blatchford score was 13 (IQR 11-15; $p<0.0001$, Kruskal-Wallis test). There was a reasonable correlation between urea level and Blatchford score, as demonstrated by a Spearman coefficient of 0.74 (95\% CI 0.66-0.80; Fig. 1) [19]. Additionally, receiver operating characteristic curves for urea level and the Blatchford score to identify a source of bleeding on EGD showed very similar results, with moderate diagnostic accuracy according to Swets' criteria [20]. The area under the curve for urea was $0.642(p<$ $0.001)$ and $0.660(p<0.001)$ for the Blatchford score (Fig. 2, 3).

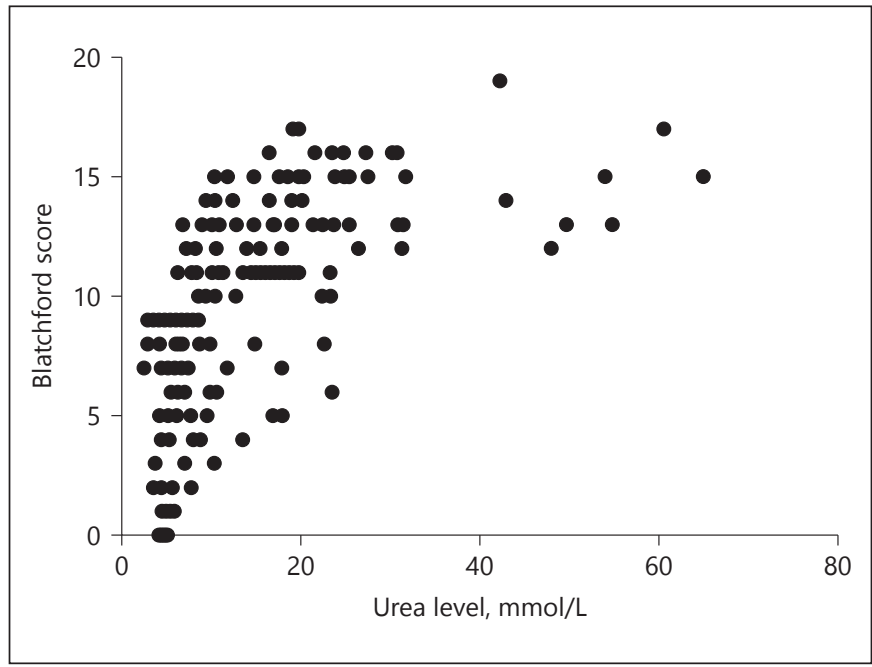

Fig. 1. Scatter plot of urea vs. Blatchford score. Spearman $R$ coefficient of 0.74 (95\% CI 0.66-0.80), $p<0.0001$. 


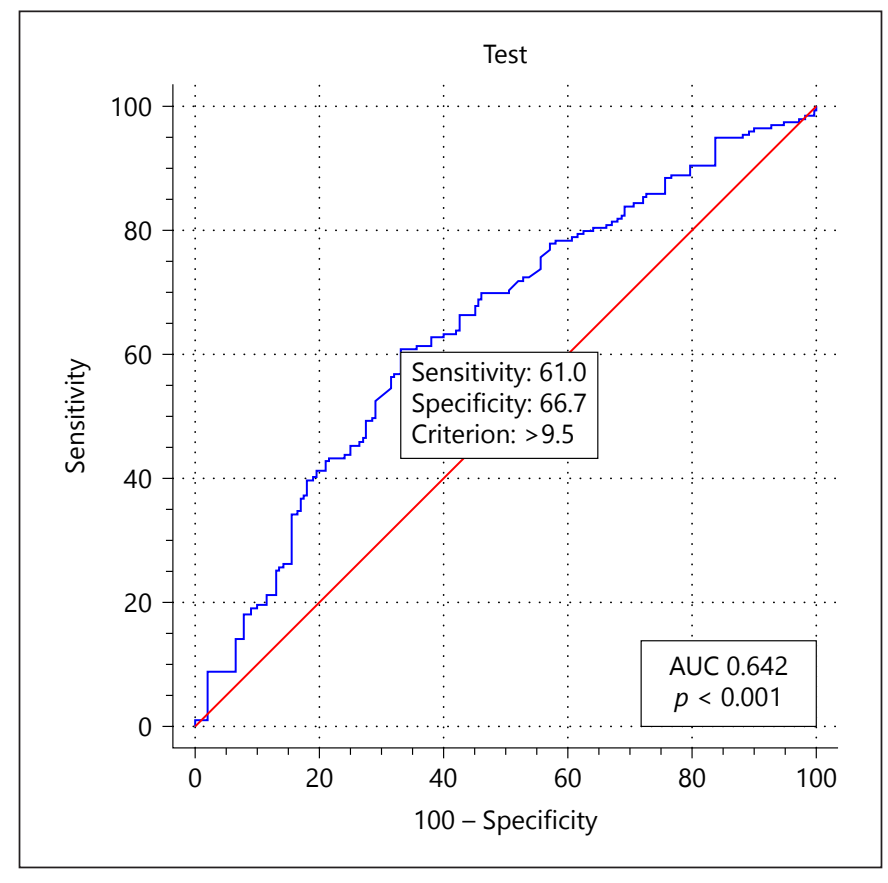

Fig. 2. Receiver operating characteristic curves analysis for urea level and source of GI bleed identified on EGD. AUC, area under the curve.

\section{Discussion}

The results of this study provide new clinically relevant information regarding the operating characteristics of urea for identifying a source of UGIB on EGD. Urea level appears to be an independent predictor of positive endoscopic findings in presumed UGIB. Given that not all patients presenting to hospital with UGIB have endoscopic evidence of culprit lesions, it is important to understand the factors which can predict positive findings. Our multivariate logistic regression model identified significantly increased odds of identifying UGIB lesions at the time of EGD in males, patients with melena, and patients with hematemesis. This is consistent with current literature and highlights the need to be aware of these signs on history, as they may be markers of a more severe UGIB [21-23].

Some studies have proposed that urea level is a weak predictor of UGIB and is not helpful in identifying high-risk lesions [18]. Subsequent literature has found that higher urea levels may correlate with UGIB severity [21]. We were able to identify a threshold level of urea above which the odds of identifying a source of UGIB at endoscopy was significantly elevated. The odds of identifying a source of UGIB at endoscopy for pa-

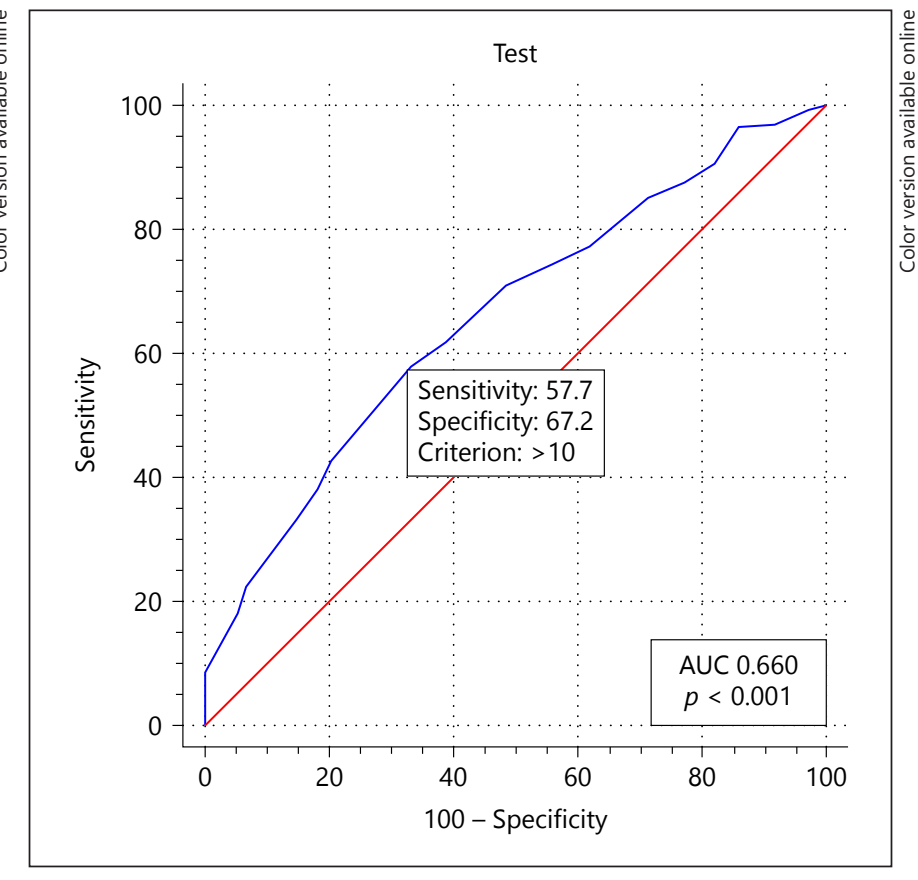

Fig. 3. Receiver operating characteristic curves analysis for Blatchford score and source of GI bleed identified on EGD. AUC, area under the curve.

tients with urea $\geq 10 \mathrm{mmol} / \mathrm{L}$ was 3.73 (95\% CI 1.90 7.31) times higher than patients with urea $<10 \mathrm{mmol} / \mathrm{L}$. In our cohort, this was one of the strongest predictors of finding a potential culprit lesion at upper GI endoscopy. Kumar et al. [24] demonstrated that even after adjusting for the AIMS65 score, an increase in urea within $24 \mathrm{~h}$ was independently correlated with a composite outcome of inpatient death, inpatient re-bleeding, need for surgical or radiologic intervention or endoscopic re-intervention [22-24]. Despite the exclusion of cirrhotic patients in the study by Kumar et al. [24], this suggests that perhaps both initial urea level and rise in urea level over time can be highly predictive of UGIB. Our study found that the PPV of urea $\geq 10 \mathrm{mmol} / \mathrm{L}$ for positive findings on EGD is $80 \%$. The PPV of Blatchford scores at various evaluated thresholds was $71-72 \%$ in our study population. This is consistent with the literature where a Blatchford score of $>2$ has a PPV of $74 \%$ for predicting the need for hospital-based intervention or 30-day mortality [25]. Hence, urea $\geq 10 \mathrm{mmol} / \mathrm{L}$ on its own certainly does have predictive value for identifying an upper GI lesion on EGD and is a much easier tool to use than calculating a complete score. Our study methodology involved selection of patients who had EGD performed for suspected bleeding, so this patient 
population has a high pre-test probability of GI bleeding. PPVs are dependent on the baseline prevalence of a disease. This implies that for a patient who has a high pre-test likelihood of gastrointestinal bleeding, urea $>10 \mathrm{mmol} / \mathrm{L}$ predicts in $80 \%$ of cases, a source of GI bleeding will be identified. These findings may not be applicable for a patient population with low pre-test likelihood of gastrointestinal bleeding.

Our study reveals that for patients with normal renal function, cirrhosis, or NSAID use, urea $\geq 10 \mathrm{mmol} / \mathrm{L}$ has a high PPV for significant source of UGIB. Of note, for patients with normal renal function, the sensitivity of urea $\geq 10 \mathrm{mmol} / \mathrm{L}$ was only $38 \%$ for identifying a source of UGIB at endoscopy. This suggests that in patients with normal renal function, a normal urea level cannot necessarily exclude a bleeding source at endoscopy. The NPV of urea $\leq 10 \mathrm{mmol} / \mathrm{L}$ for identifying a high-risk bleeding source (varices, high-risk ulcer, Dieulafoy, aortoenteric fistula) or the use of EGD intervention was 90 and 85\%, respectively. These features imply that urea level prior to endoscopy can be greatly informative to the clinician and may help better triage patients presenting with presumed UGIB.

This study validates that the Blatchford score is highly sensitive for predicting UGIB $[12,17]$. A good correlation between Blatchford score and urea level was also demonstrated (Spearman coefficient of 0.74), suggesting that urea may be used as a surrogate marker for the severity of UGIB. These tests may have different utilities. Whereas a Blatchford score of 0 is useful for ruling out UGIB and can provide the impetus to more confidently discharge lowrisk patients, a patient with suspected UGIB and an ele- vated urea strongly predicts identifying a source of UGIB at EGD.

Some limitations of this study include the retrospective nature and inability to control for unknown confounders. For example, we did not have access to information regarding patient volume status or urine output and could not address hydration status in this population, hence dehydration could be a potential confounder. Our study may be underpowered to look at the association of certain variables like ICU admission or presence of cirrhosis, which explains the wide confidence intervals seen for some variables in Table 2 . The variability in timing at which upper endoscopy was conducted in relation to patient admission could potentially also affect the likelihood of positive findings on EGD, however, this period would likely not have been long enough to allow for complete healing of lesions, such as NSAID ulcers or erosions. We acknowledge that the use of lowest hemoglobin level prior to endoscopy may have been affected by dilution from intravenous fluids, in addition to GI bleeding.

Overall, urea level is an independent predictor of positive endoscopic findings in presumed UGIB and urea 10 $\mathrm{mmol} / \mathrm{L}$ may be a useful clinical threshold for physicians to consider endoscopic evaluation to identify a bleeding source. Future prediction models for UGIB should consider incorporation of urea as a prognostic variable given the findings of this study.

\section{Disclosure Statement}

The authors declare that they have no conflicts of interest.

\section{References}

1 Leontiadis GI, Sreedharan A, Dorward S, Barton P, Delaney B, Howden CW, et al. Systematic reviews of the clinical effectiveness and cost-effectiveness of proton pump inhibitors in acute upper gastrointestinal bleeding. Health Technol Assess. 2007 Dec;11(51):iiiiv.

2 Rockall TA, Logan RF, Devlin HB, Northfield TC. Incidence of and mortality from acute upper gastrointestinal haemorrhage in the United Kingdom. Steering Committee and members of the National Audit of Acute Upper Gastrointestinal Haemorrhage. BMJ. 1995 Jul;311(6999):222-6.

3 Blatchford O, Davidson LA, Murray WR, Blatchford M, Pell J. Acute upper gastrointestinal haemorrhage in west of Scotland: case ascertainment study. BMJ. 1997 Aug; 315(7107):510-4.
4 El-Tawil AM. Trends on gastrointestinal bleeding and mortality: wherearewestanding? World J Gastroenterol. 2012 Mar;18(11):1154-8.

5 Gilbert DA. Epidemiology of upper gastrointestinal bleeding. Gastrointest Endosc. 1990 Sep-Oct;36(5 suppl):S8-13.

6 Laine LA. Helicobacter pylori and complicated ulcer disease. Am J Med. 1996 May; 100(5A):52S-7S

7 Sharara AI, Rockey DC. Gastroesophageal variceal hemorrhage. N Engl J Med. 2001 Aug;345(9):669-81.

8 Grace ND, Groszmann RJ, Garcia-Tsao G, Burroughs AK, Pagliaro L, Makuch RW, et al. Portal hypertension and variceal bleeding: an AASLD single topic symposium. Hepatology. 1998 Sep;28(3):868-80.

9 Chak A, Cooper GS, Lloyd LE, Kolz CS, Barnhart BA, Wong RC. Effectiveness of endos- copy in patients admitted to the intensive care unit with upper GI hemorrhage. Gastrointest Endosc. 2001 Jan;53(1):6-13.

10 Lin HJ, Wang K, Perng CL, Chua RT, Lee FY, Lee $\mathrm{CH}$, et al. Early or delayed endoscopy for patients with peptic ulcer bleeding. A prospective randomized study. J Clin Gastroenterol. 1996 Jun;22(4):267-71.

11 Hwang JH, Fisher DA, Ben-Menachem T, Chandrasekhara V, Chathadi K, Decker GA, et al.; Standards of Practice Committee of the American Society for Gastrointestinal Endoscopy. The role of endoscopy in the management of acute non-variceal upper GI bleeding. Gastrointest Endosc. 2012 Jun;75(6):1132-8.

12 Blatchford O, Murray WR, Blatchford M. A risk score to predict need for treatment for upper-gastrointestinal haemorrhage. Lancet. 2000 Oct;356(9238):1318-21.
Urea Concentration and Positive Endoscopic Findings
Dig Dis 2020;38:77-84

DOI: $10.1159 / 000501549$ 
13 Barkun AN, Bardou M, Kuipers EJ, Sung J, Hunt RH, Martel M, et al.; International Consensus Upper Gastrointestinal Bleeding Conference Group. International consensus recommendations on the management of patients with nonvariceal upper gastrointestinal bleeding. Ann Intern Med. 2010 Jan;152(2):101-13.

14 Chen IC, Hung MS, Chiu TF, Chen JC, Hsiao CT. Risk scoring systems to predict need for clinical intervention for patients with nonvariceal upper gastrointestinal tract bleeding. Am J Emerg Med. 2007 Sep;25(7):774-9.

15 Srirajaskanthan R, Conn R, Bulwer C, Irving $P$. The Glasgow Blatchford scoring system enables accurate risk stratification of patients with upper gastrointestinal haemorrhage. Int J Clin Pract. 2010 Jun;64(7):868-74.

16 Stanley AJ, Ashley D, Dalton HR, Mowat C, Gaya DR, Thompson E, et al. Outpatient management of patients with low-risk uppergastrointestinal haemorrhage: multicentre validation and prospective evaluation. Lancet. 2009 Jan;373(9657):42-7.
17 Masaoka T, Suzuki H, Hori S, Aikawa N, Hibi T. Blatchford scoring system is a useful scoring system for detecting patients with upper gastrointestinal bleeding who do not need endoscopic intervention. J Gastroenterol Hepatol. 2007 Sep;22(9): 1404-8.

18 Al-Naamani K, Alzadjali N, Barkun AN, Fallone CA. Does blood urea nitrogen level predict severity and high-risk endoscopic lesions in patients with nonvariceal upper gastrointestinal bleeding? Can J Gastroenterol. 2008 Apr;22(4):399-403.

19 Mukaka MM. Statistics corner: A guide to appropriate use of correlation coefficient in medical research. Malawi Med J. 2012 Sep; 24(3):69-71.

20 Swets JA. ROC analysis applied to the evaluation of medical imaging techniques. Invest Radiol. 1979 Mar-Apr;14(2):109-21.

21 Tomizawa M, Shinozaki F, Hasegawa R, Shirai Y, Motoyoshi Y, Sugiyama T, et al. Patient characteristics with high or low blood urea ni- trogen in upper gastrointestinal bleeding. World J Gastroenterol. 2015 Jun;21(24): 7500-5.

22 Chung IK. Predictive factors for endoscopic hemostasis in patients with upper gastrointestinal bleeding. Clin Endosc. 2014 Mar;47(2): 121-3.

23 Srygley FD, Gerardo CJ, Tran T, Fisher DA. Does this patient have a severe upper gastrointestinal bleed? JAMA. 2012 Mar;307(10): 1072-9.

24 Kumar NL, Claggett BL, Cohen AJ, Nayor J, Saltzman JR. Association between an increase in blood urea nitrogen at 24 hours and worse outcomes in acute nonvariceal upper GI bleeding. Gastrointest Endosc. 2017 Dec;86(6):1022-1027.e1.

25 Laursen SB, Hansen JM, Schaffalitzky de Muckadell OB. The Glasgow Blatchford score is the most accurate assessment of patients with upper gastrointestinal hemorrhage. Clin Gastroenterol Hepatol. 2012 Oct;10(10): 1130-1135.e1. 